\title{
Sub-THz Characterisation of Monolayer Graphene
}

\author{
Ehsan Dadrasnia, ${ }^{1}$ Sujitha Puthukodan, ${ }^{1}$ \\ Vinod V. K. Thalakkatukalathil, ${ }^{1}$ Horacio Lamela, ${ }^{1}$ Guillaume Ducournau, ${ }^{2}$ \\ Jean-Francois Lampin, ${ }^{2}$ Frédéric Garet, ${ }^{3}$ and Jean-Louis Coutaz ${ }^{3}$ \\ ${ }^{1}$ Optoelectronics and Laser Technology Group (GOTL), Carlos III de Madrid University, 28911 Leganes, Madrid, Spain \\ ${ }^{2}$ IEMN, UMR CNRS 8520, University of Lille, Avenue Poincaré, BP 60069, 59652 Villeneuve d'Ascq, France \\ ${ }^{3}$ IMEP-LAHC, UMR CNRS 5130, University of Savoie, 73376 Le Bourget du Lac Cedex, France
}

Correspondence should be addressed to Horacio Lamela; horacio@ing.uc3m.es

Received 14 December 2013; Accepted 12 May 2014; Published 7 July 2014

Academic Editor: Eugen Culea

Copyright ( 2014 Ehsan Dadrasnia et al. This is an open access article distributed under the Creative Commons Attribution License, which permits unrestricted use, distribution, and reproduction in any medium, provided the original work is properly cited.

We explore the optical and electrical characteristics of monolayer graphene by using pulsed optoelectronic terahertz time-domain spectroscopy in the frequency range of $325-500 \mathrm{GHz}$ based on fast direct measurements of phase and amplitude. We also show that these parameters can, however, be measured with higher resolution using a free space continuous wave measurement technique associated with a vector network analyzer that offers a good dynamic range. All the scattering parameters (both magnitude and phase) are measured simultaneously. The Nicholson-Ross-Weir method is implemented to extract the monolayer graphene parameters at the aforementioned frequency range.

\section{Introduction}

One-dimensional (1D) carbon nanotubes (CNTs) and twodimensional (2D) graphene forms of carbon nanostructures are considered as alternative materials to indium-tin oxide (ITO) for flexible transparent conductive films, since they have unique electrical, optical, thermal, and mechanical properties [1-5]. Carbon nanostructures have been applied in electronic devices, such as conductive flexible adhesives [6] and field-effect transistors [7]. The high mobility of electrons [8] and optical conductivity [9] in graphene have been experimentally investigated to study the ultrafast intra-/interband transitions of two-dimensional Dirac fermions and ballistic transport at room temperature $[10,11]$. Contact devices have been employed to characterize the current voltage of metallic and semiconducting carbon nanostructure samples and to determine their remarkable electrical properties $[12,13]$.

Many materials that are opaque to visible and infrared light are transparent to terahertz $(\mathrm{THz})$ radiation. Moreover, microwave and far infrared (IR) absorption spectroscopy have limitations because of the complicated numerical transformation process. The nondestructive and noncontact optoelectronic setups that generate and detect coherent $\mathrm{THz}$ electromagnetic waves facilitate to characterize both thin and bulk materials. The advantages of continuous wave (CW) characterization using vector network analyzer (VNA) over pulsed terahertz time-domain spectroscopy (THz-TDS) are higher spectral resolution and huge dynamic range in the sub-THz frequencies $(60-70 \mathrm{~dB}$ around $500 \mathrm{GHz})$ [14]. For above $500 \mathrm{GHz}$, pulsed THz-TDS offers better spectral resolution and dynamic range. Electrical properties, such as conductivity, can be easily derived from the measured optical constants at THz frequencies $[15,16]$.

It was believed that the synthesis of a single sheet graphene was difficult. But in 2004, Novoselov and Geim showed that it is possible to isolate monolayer graphene and to deposit it on a substrate and that the electrical and optical measurements can be performed $[17,18]$. Here we present the experimental study of MG deposited on a fused quartz substrate in the frequency range of $325-500 \mathrm{GHz}$ by 
using both pulsed and CW techniques at room temperature. The optical and electrical properties are studied using $\mathrm{THz}-$ TDS and it is compared with CW-VNA technique since it offers a higher spectral resolution and a good dynamic range especially at sub- $\mathrm{THz}$ frequencies. The synthesis of MG sample and its Raman spectral properties are given in Section 2. The experimental setups and the parameter extractions are explained in Section 3. The obtained results are discussed in Section 4.

\section{Monolayer Graphene Sample}

MG sample was purchased from Meijo Nano Carbon Ltd (Japan). It was synthesized through chemical vapor deposition (CVD) reaction after the epitaxial growth of metal catalysts on a substrate which is then transferred onto a fused quartz base $\left(20 \times 20 \times 0.49 \mathrm{~mm}^{3}\right)$. The fused quartz substrate was chosen to facilitate the detection of $\mathrm{THz}$ waves to reduce the attenuation of the propagated beam.

The number of layers and the electronic structure of graphene sample can be explored in Raman spectrum [19]. Number of graphene layers and quality of fabricated sample can change the intensity of $G$ and $2 D$ peaks [20]. Figure 1 shows the Raman spectra of transferred MG taken under this study with $\mathrm{G}\left(\sim 1580 \mathrm{~cm}^{-1}\right)$ and $2 \mathrm{D}\left(\sim 2680 \mathrm{~cm}^{-1}\right)$ bands. The intensity of disorder-induced $\mathrm{D}$ band $\left(\sim 1,350 \mathrm{~cm}^{-1}\right)$ may be because of edges and subdomain boundaries in graphene consistent with MG as discussed in [21] with intensity $I_{\mathrm{D}} / I_{\mathrm{G}}$ ratio $\sim 0.2$.

\section{Sub-THz Experimental Setups}

3.1. Pulsed Time-Domain THz Spectroscopy. We perform the $\mathrm{THz}$ characterization of the described MG sample using a conventional THz-TDS setup as shown in Figure 2 [22, 23]. The samples are located at the waist of the $\mathrm{THz}$ beam that can be considered as an almost perfect Gaussian beam, whose section is very much smaller than the sample surface 20 $\times 20 \mathrm{~mm}^{2}$. Hence, no diaphragm is needed to resize the $\mathrm{THz}$ beam to pass through the sample. Moreover, we can notice that the Rayleigh length of the $\mathrm{THz}$ beam is much longer than the sample thickness throughout the studied $\mathrm{THz}$ frequency range. Therefore, the samples can be considered as illuminated by a plane $\mathrm{THz}$ wave. The waveforms are recorded over a 35 ps time-window, leading to a spectral resolution of $28 \mathrm{GHz}$.

Three measurements of temporal waveforms are performed. At first, the reference signal for air is recorded. Secondly, the quartz substrate is placed between the $\mathrm{THz}$ emitter and detector antennas and the corresponding reference signal is recorded. The reference substrate is then replaced by the sample and once again the measurement is performed. For each sample, the measurements are repeated four times to find the average amplitude of each $\mathrm{THz}$ waveform. The Fourier transform technique is used to map the $\mathrm{THz}$ spectrum.

The field transmitted through the MG takes a more complicated expression than reference substrate. Thus, the extraction procedure to determine the complex refractive index of the MG is difficult, as it takes a complex value because of the losses caused by it. Since the substrate thickness $D$ is much higher than the thickness of the MG, one can time-window the recorded waveform in order to get rid of the temporal echoes that reflect back and forth in the substrate [24]. In the frequency domain, the expression of the three Fourier-transformed measured signals for reference air $\left(S_{R}\right)$, the bare substrate $\left(S_{S}\right)$, and the substrate covered with $\mathrm{MG}\left(S_{f}\right)$ can be written as [24-26]

$$
\begin{gathered}
S_{R}(\omega)=S_{o} e^{j(\omega / c) d} e^{j(\omega / c) D}, \\
S_{S}(\omega)=S_{o} t_{a s} t_{s a} e^{j(\omega / c) d} e^{j(\omega / c) \widetilde{n}_{s} D}, \\
S_{F}(\omega)=S_{o} \frac{t_{a f} t_{f s} t_{s a}}{1-r_{f s} r_{f a} e^{j 2(\omega / c) d \widetilde{n}_{f}}} e^{j(\omega / c) d \widetilde{n}_{f}} e^{j(\omega / c) \widetilde{n}_{s} D} .
\end{gathered}
$$

The subscripts $a, f$, and $s$ are for ambient medium (air), MG sample, and substrate. $S_{o}$ is the air-incident beam. $d$ is the thickness of the MG sample and $t_{i j}$ and $r_{i j}$ are the coefficients of transmission and reflection at the interface between media $i$ and $j . \widetilde{n}_{i}=n_{i}+j \kappa_{i}$ is the complex refractive index of medium $i$. Then, $n_{s}$ and $n_{f}$ are the refractive index of the bare substrate and the MG sample, respectively. To derive (2) and (3), we made the hypothesis that the substrate is thick enough to get rid of the rebounds of the $\mathrm{THz}$ pulses occurring inside by an appropriate time-windowing. From (1) and (2), we determine the refractive index $\widetilde{n}_{s}$ of the substrate using a classical THzTDS extraction procedure. From (2) and (3), the complex transmission $T(\omega)$ for thin film with magnitude $(\rho)$ and phase shift $(\phi)$ can be defined as

$$
T(\omega)=\frac{S_{F}(\omega)}{S_{S}(\omega)}=\rho(\omega) e^{i \phi(\omega)} .
$$

As the thickness of $\mathrm{MG}\left(d_{f}\right)$ is nanometric and also $j(\omega / c)=$ $\left(Z_{0} \sigma_{f} / \widetilde{n}_{f}^{2}\right)$ for a highly conductive film, we can take an approximation of $\left(\widetilde{n}_{s} / \widetilde{n}_{f}^{2}\right) \ll 1$ and (4) can be written as

$$
T(\omega) \approx \frac{1+n_{s}(\omega)}{1+n_{s}(\omega)+Z_{0} d \sigma_{f}(\omega)},
$$

where $\sigma_{f}=\sigma_{f, r}+j \sigma_{f, i m}=j \omega \varepsilon_{o} \varepsilon_{f}$ is complex conductivity of the MG.

\subsection{CW Sub-THz Measurement Using a Vector Network} Analyzer. The schematic diagram of the experimental setup for sub-THz characterization of the $\mathrm{MG}$ sample is given in Figure 3. The measurement is carried out in free space using a Rohde and Schwarz ZVA 24 VNA associated with frequency extenders. The transmitting and receiving ports are connected to rectangular waveguides which act as antennas. The sample is placed between these waveguides with the help of a sample holder. The $\mathrm{THz}$ beam is manipulated using two polymer lenses and the sample is placed in the collimated part of the beam.

The VNA simultaneously delivers both magnitude and phase of scattering $(S)$ parameters. The measurement is 


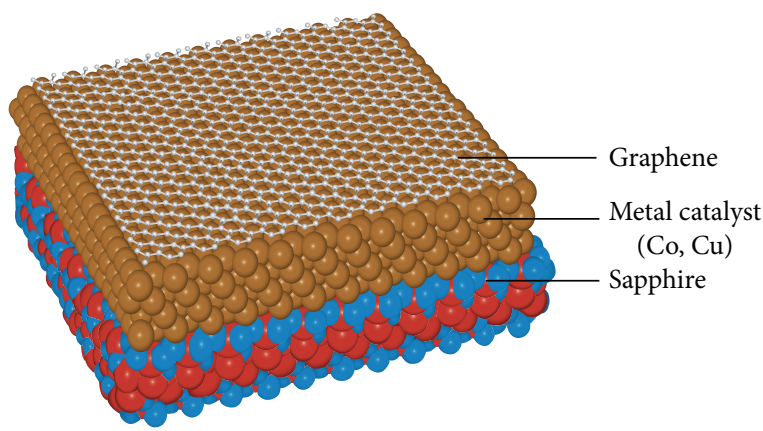

(a)

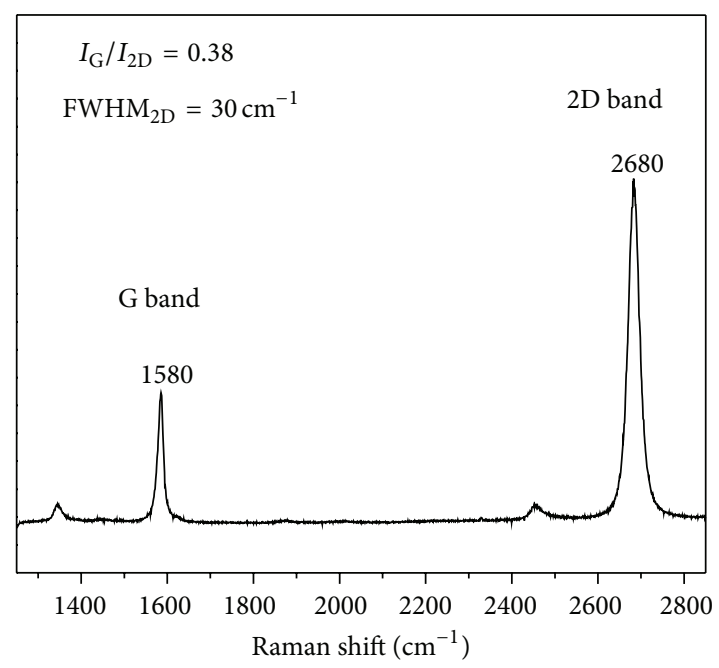

(b)

FIGURE 1: Model structure of transferred monolayer graphene (a). Raman spectra of transferred single layered graphene with G and 2D peaks (b) (Meijo Nano Carbon Ltd.).

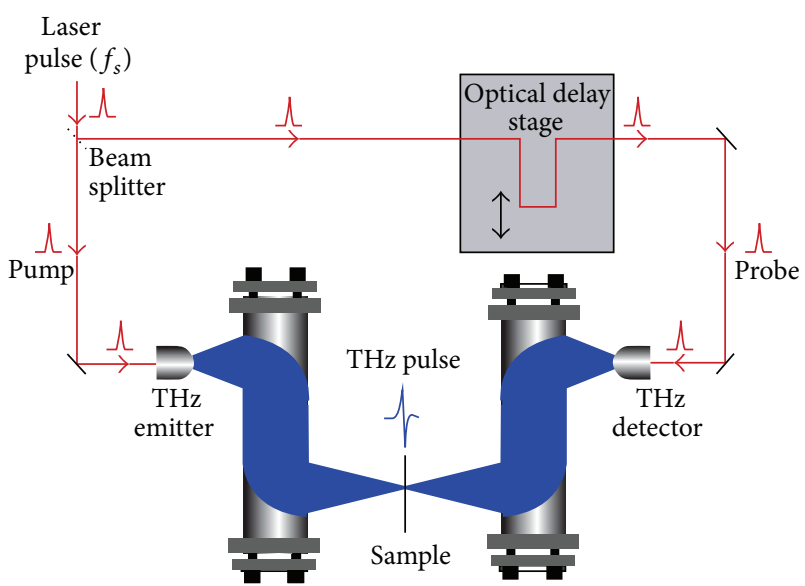

FIGURE 2: Schematic diagram of the THz-TDS setup.

done with a $0.525 \mathrm{GHz}$ spectral resolution. The maximum frequency of the VNA is $24 \mathrm{GHz}$ which derives the extenders. But at the extender output, as the signal is multiplied inside, the maximum operating frequency is increased up to $500 \mathrm{GHz}$. The system is first calibrated using the TRM (through, reflect, and match) method before starting the sample measurements. Once calibrated, the reference measurements are done for a bare substrate [27]. The calibration frequency is direct in the $\mathrm{THz}$ range as we use a waveguide calibration in range $325-500 \mathrm{GHz}$ with a step of about $500 \mathrm{MHz}$. This step is sufficient as no fast frequency variation is expected for the measured sample.

The normally incident beam is allowed to pass through the sample. The corresponding $S$-parameters are recorded. The measurements are once again performed for the MG

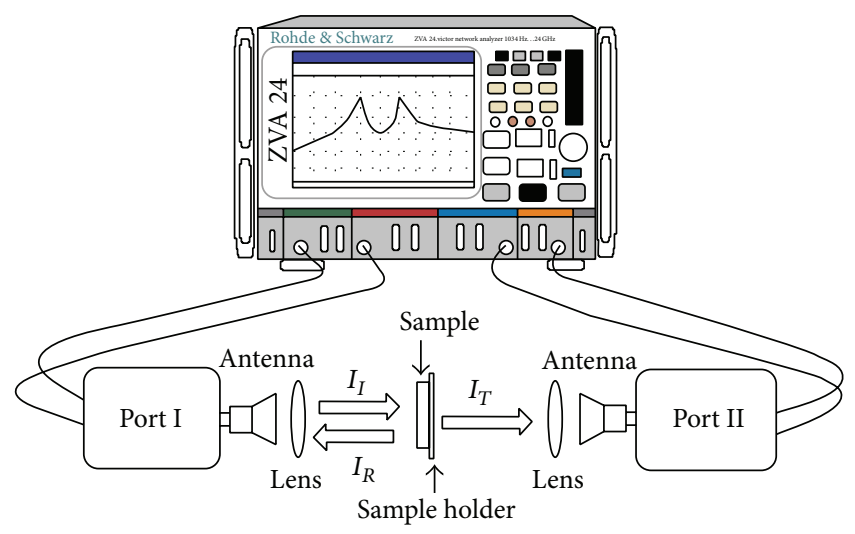

FIGURE 3: Schematic diagram of CW free space measurement experimental setup.

deposited on the fused quartz substrate. To extract the refractive index and conductivity of the MG sample alone, we have first removed the effect of substrate [28, 29]. The material parameters of the MG are extracted by implementing the Nicholson-Ross-Weir (NRW) method [30, 31]. According to NRW method [32,33], the $S$-parameters can be expressed in terms of reflection coefficient and transmission coefficient as

$$
\begin{aligned}
& S_{21}=\frac{\left(1-R^{2}\right) T}{1-R^{2} T^{2}}, \\
& S_{11}=\frac{\left(1-T^{2}\right) R}{1-R^{2} T^{2}},
\end{aligned}
$$

where $T=e^{-\gamma d}$ and $R=\left(\mu \gamma_{0}-\gamma\right) /\left(\mu \gamma_{0}+\gamma\right)$ are the transmission and reflection coefficients, respectively. $\gamma_{0}=$ 


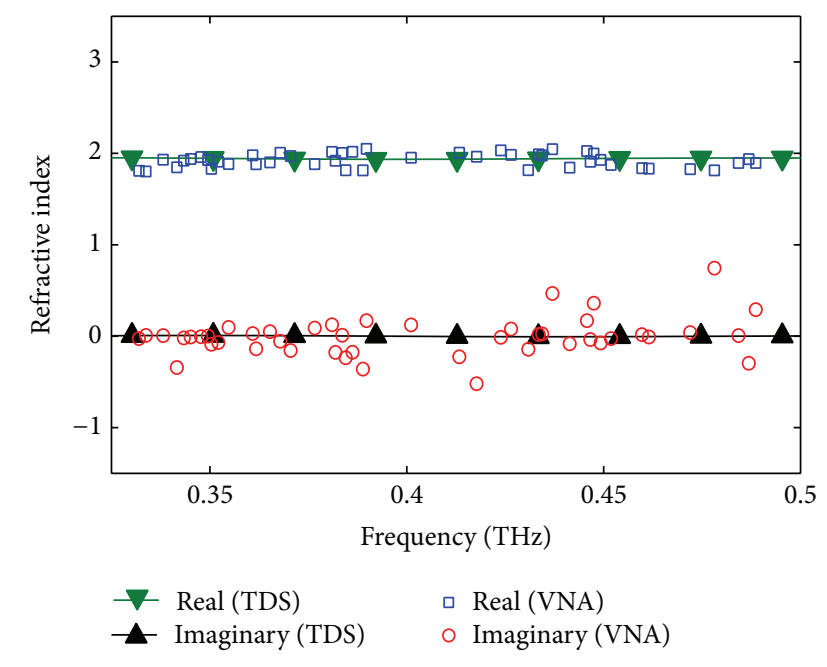

FIgURE 4: The complex refractive index of fused quartz as a function of frequency for both (i) THz TDS and (ii) CW-VNA methods.

$\sqrt{k_{c}{ }^{2}-k_{0}^{2}}$ is the free space propagation constant. The cutoff wavenumber $k_{c}=\pi / a$, where $a$ is the longer dimension of the rectangular waveguide and $k_{0}$ is the wavenumber in free space. The propagation constant can be expressed as

$$
\gamma=\frac{\ln |1 / T|+j[\arg (1 / T)+2 m \pi]}{d},
$$

where $m$ is an integer. Since $T$ is complex, the function $\ln |1 / T|$ has an infinite number of roots. Once the propagation constant $\gamma$ is obtained, the effective permittivity of the sample is derived as follows:

$$
\varepsilon_{f}=\varepsilon_{1}-i \varepsilon_{2}=\frac{k_{c}^{2}-\gamma^{2}}{\mu k_{0}^{2}} .
$$

The refractive index and conductivity are calculated from the extracted permittivity.

\section{Results and Discussion}

The optical and electrical properties of the reference substrate are first extracted followed by a parameter extraction of MG sample (Section 3). We determine the complex refractive index of the substrate using a classical THz-TDS extraction procedure [24] and the NRW method. The fused quartz substrate for both noncovered and covered samples with a thickness of $0.49 \mathrm{~mm}$ has a refractive index $\left(n_{s}\right) \sim 1.95$. The extinction coefficient $k_{s}$ of the substrate is nearly zero in the frequency range of $325-500 \mathrm{GHz}$ (Figure 4). And the absorption $\alpha=2 k_{s} \omega / c$ increases continuously with frequency to reach $8 \mathrm{~cm}^{-1}$ at $2 \mathrm{THz}$ [22]. In CW results, the measurements are not averaged as curves fluctuations are due to Fabry-Perot residual effects, which cannot be suppressed with averaging.
The plasma frequency and carrier density of MG have a nonlinear dependency, because of the conical dispersion. The two processes involved in optical absorption in graphene are carrier intraband transitions and interband transitions [34]. It is important to note that the intraband transition conductivity in the $\mathrm{THz}$ range dominates the interband transition conductivity [35]. The measured intraband conductivity is 30 times larger than that of the interband [36]. Because of this, we only consider the conductivity by the intraband free-carrier response in sub- $\mathrm{THz}$ analysis.

The THz-TDS setup may not offer enough signal-to-noise ratio to determine the precise conductivity at low frequencies, because of its sensitivity to the thickness of the MG sample. Figure 5(a) shows the frequency-dependent refractive index obtained for MG sample which shows a similar response as that of thin films with metallic behavior [37]. The high absorption of electromagnetic wave passing through the sample, shown in Figure 5(b), is in turn proportional to the carrier density of MG. Figure 5(c) shows the conductivity $\left(\sigma_{f, r}=\varepsilon_{2} \varepsilon_{0} \omega\right)$ obtained for the MG sample using THz-TDS and CW-VNA techniques.

The thickness of MG is very small compared to the thickness of the substrate which has a lower attenuation at sub-THz frequencies. Because of this, in TDS analysis, the multiple-reflections happening inside the sample are taken into account as mentioned in (Section 3.1). The values of conductivity in TDS measurement are sensitive to the thickness difference between the MG and reference substrate. CW-VNA method offers a higher spectral resolution and a good dynamic range. As seen, the obtained results using TDS analysis and NRW method are consistent with respect to each other.

The highly conductive and flexible nature of graphene promises it as a material for $\mathrm{THz}$ based applications such as broadband optoelectronic modulators [34, 35] and fieldeffect transistors [38].

\section{Conclusion}

In this paper, two different techniques have been demonstrated as powerful noncontact nondestructive tools to study the optical and electrical properties of MG sample deposited on fused quartz substrate in the sub- $\mathrm{THz}$ frequency ranges of $325-500 \mathrm{GHz}$. Using THz-TDS measurement, we have considered the surface boundary condition of MG sample between transparent fused quartz substrate and air to precisely achieve the complex refractive index and electrical conductivity of single-layer graphene. We have also studied the MG sample using CW-VNA free space technique. The NRW approach has been utilized in order to extract the material parameters. The main advantages of using a VNA are higher spectral resolution and a good dynamic range in sub-THz frequencies.

In conclusion, it can be seen that the high absorption and conductive behavior of graphene can be studied using pulsed and CW Sub-THz techniques which promise a nondestructive characterization of nanoscale samples. 


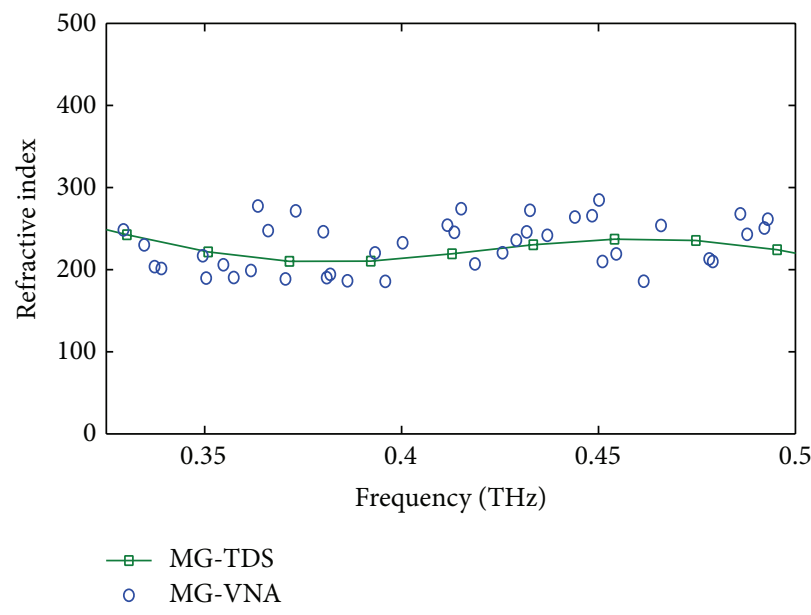

(a)

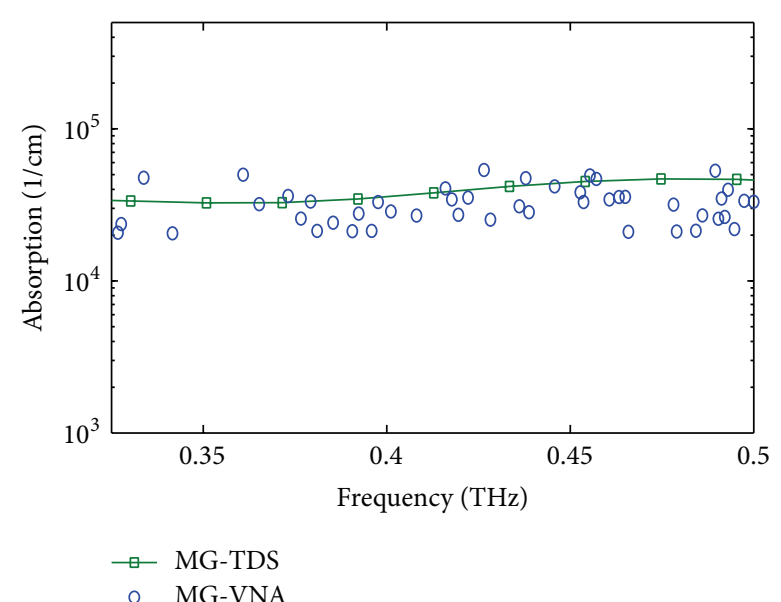

(b)

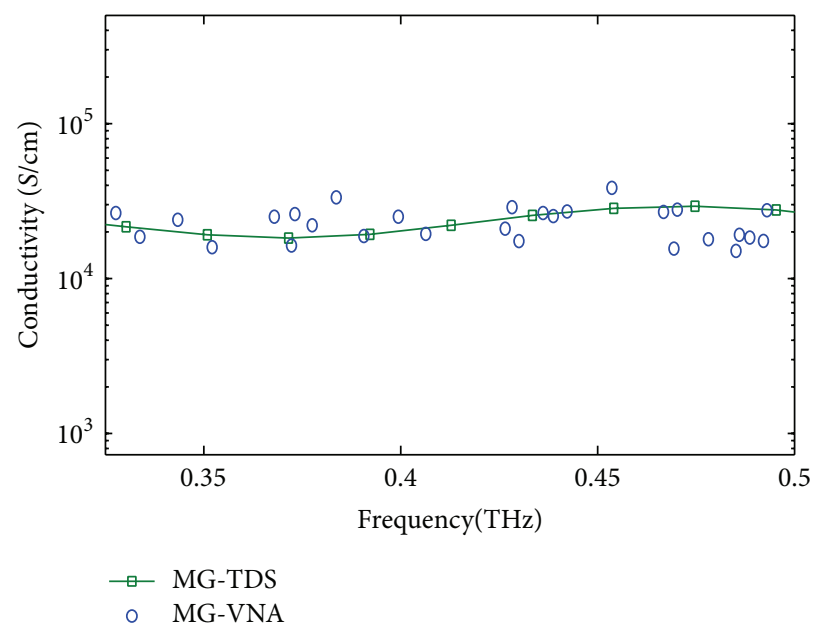

(c)

FIGURE 5: The obtained (a) real refractive index, (b) power absorption, and (c) electrical conductivity of MG using both THz-TDS and CW NRW techniques.

\section{Conflict of Interests}

The authors declare that there is no conflict of interests regarding the publication of this paper.

\section{Acknowledgment}

This work was supported by the project MITEPHO (Microwave and Terahertz Photonics, ITN-FP7, 238393) under the European Commission, which seeks to investigate and develop compact and integrated tunable dual-mode diode lasers for $\mathrm{THz}$ spectroscopy in sensing, nanotechnology, and biomedical applications.

\section{References}

[1] Z. Wu, Z. Chen, X. Du et al., "Transparent, conductive carbon nanotube films," Science, vol. 305, pp. 1273-1276, 2004.
[2] S. Bae, H. Kim, Y. Lee et al., "Roll-to-roll production of 30-inch graphene films for transparent electrodes," Nature Nanotechnology, vol. 5, pp. 574-578, 2010.

[3] P. Bandaru, "Electrical properties and applications of carbon nanotube structures," Journal of Nanoscience and Nanotechnology, vol. 7, pp. 1239-1267, 2007.

[4] A. A. Balandin, "Thermal properties of graphene and nanostructured carbon materials," Nature Materials, vol. 10, pp. 569581, 2011.

[5] K. M. F. Shahil and A. A. Balandin, "Thermal properties of graphene and multilayer graphene: applications in thermal interface materials," Solid State Communications, vol. 152, pp. 1331-1340, 2012.

[6] R. Ma, S. Kwon, Q. Zheng et al., "Carbon-nanotube/silver networks in nitrile butadiene rubber for highly conductive flexible adhesives," Advanced Materials, vol. 24, no. 25, pp. 3344-3349, 2012.

[7] H. R. Byon and H. C. Choi, "Network single-walled carbon nanotube-field effect transistors (SWNT-FETs) with increased 
Schottky contact area for highly sensitive biosensor applications," Journal of the American Chemical Society, vol. 128, pp. 2188-2189, 2006.

[8] A. H. Castro Neto, F. Guinea, N. M. R. Peres, K. S. Novoselov, and A. K. Geim, "The electronic properties of graphene," Reviews of Modern Physics, vol. 81, p. 109, 2009.

[9] K. F. Mak, M. Y. Sfeir, Y. Wu, C. H. Lui, J. A. Misewich, and T. F. Heinz, "Measurement of the optical conductivity of graphene," Physical Review Letters, vol. 101, Article ID 196405, 2008.

[10] H. Choi, F. Borondics, D. A. Siegel et al., "Broadband electromagnetic response and ultrafast dynamics of few-layer epitaxial graphene," Applied Physics Letters, vol. 94, Article ID 172102, 2009.

[11] V. Ryzhii, M. Ryzhii, and T. Otsuji, "Negative dynamic conductivity of graphene with optical pumping," Journal of Applied Physics, vol. 101, Article ID 083114, 2007.

[12] T. W. Ebbesen, H. J. Lezec, H. Hiura, J. W. Bennett, H. F. Ghaemi, and T. Thio, "Electrical conductivity of individual carbon nanotubes," Nature, vol. 382, pp. 54-56, 1996.

[13] P. L. McEuen and J.-Y. Park, "Electron transport in single-walled carbon nanotubes," MRS Bulletin, vol. 29, no. 4, pp. 272-275, 2004.

[14] B. Yang, H. Su, and R. S. Donnan, "Vector network analysis of dielectric and magnetic materials in millimeter wave band," Journal of Physics: Conference Series, vol. 286, Article ID 012019, 2011.

[15] M. Naftaly and R. E. Miles, "Terahertz time-domain spectroscopy for material characterization," Proceedings of the IEEE, vol. 95, pp. 1658-1665, 2007.

[16] P. U. Jepsen, D. G. Cooke, and M. Koch, "Terahertz spectroscopy and imaging-modern techniques and applications," Laser \& Photonics Reviews, vol. 5, pp. 124-166, 2011.

[17] K. S. Novoselov, A. K. Geim, S. V. Morozov et al., "Electric field effect in atomically thin carbon films," Science, vol. 306, pp. 666669, 2004.

[18] K. S. Novoselov, D. Jiang, F. Schedin et al., "Two-dimensional atomic crystals," Proceedings of the National Academy of Sciences of the United States of America, vol. 102, no. 30, pp. 10451-10453, 2005.

[19] A. C. Ferrari, J. C. Meyer, V. Scardaci et al., "Raman spectrum of graphene and graphene Layers," Physical Review Letters, vol. 97, Article ID 187401, 2006.

[20] I. Calizo, I. Bejenari, M. Rahman, G. Liu, and A. A. Balandin, "Ultraviolet Raman microscopy of single and multilayer graphene," Journal of Applied Physics, vol. 106, Article ID 043509, 2009.

[21] L. M. Malard, M. A. Pimenta, G. Dresselhaus, and M. S. Dresselhaus, "Raman spectroscopy in graphene," Physics Reports, vol. 473, pp. 51-87, 2009.

[22] H. Lamela, E. Dadrasnia, D.-M. Lee et al., "Terahertz conductivity studies in carbon nanotube networks prepared by the vacuum filtration method," in Carbon Nanotubes, Graphene, and Associated Devices V, vol. 8462 of Proceedings of SPIE, 2012.

[23] E. Dadrasnia, S. Puthukodan, and H. Lamela, "Terahertz electrical conductivity and optical characterization of composite nonaligned single- and multiwalled carbon nanotubes," Journal of Nanophotonics, vol. 8, Article ID 083099, 2014.

[24] L. Duvillaret, F. Garet, and J. L. Coutaz, "A reliable method for extraction of material parameters in terahertz time-domain spectroscopy," IEEE Journal of Selected Topics in Quantum Electronics, vol. 2, no. 3, pp. 739-746, 1996.
[25] M. Born and E. Wolf, Principles of Optics, Cambridge University Press, Cambridge, UK, 1999.

[26] E. Dadrasnia, H. Lamela, M. B. Kuppam, F. Garet, and J.-L. Coutaz, "Determination of the DC electrical conductivity of multi-walled carbon nanotube films and graphene layers from non-contact time-domain terahertz measurements," Advances in Condensed Matter Physics, vol. 2014, Article ID 370619, 6 pages, 2014.

[27] S. Puthukodan, E. Dadrasnia, V. K. T. Vinod et al., "Sub-THz characterisation of multi-walled carbon nanotube thin films using vector network analyser," Electronics Letters, vol. 50, no. 4, pp. 297-299, 2014.

[28] L. F. Chen, C. K. Ong, C. P. Neo, V. V. Varadan, and V. K. Varadan, Microwave Electronics: Measurement and Material Characterization, John Wiley \& Sons, 2004.

[29] Z. Awang, F. A. M. Zaki, N. H. Baba, A. S. Zoolfakar, and R. A. Bakar, "A free-space method for complex permittivity measurement of bulk and thin film di-electrics at microwave frequencies," Progress in Electromagnetics Research B, vol. 51, pp. 307-328, 2013.

[30] A. Katsounaros, K. Z. Rajab, Y. Hao, M. Mann, and W. I. Milne, "Microwave characterization of vertically aligned multiwalled carbon nanotube arrays," Applied Physics Letters, vol. 98, no. 20, Article ID 203105, 2011.

[31] A. Katsounaros, K. Z. Rajab, H. Yang, M. Mann, and W. I. Milne, "Microwave characterization of multi-walled carbon nanotube arrays," in Proceedings of the 41st European Microwave Conference (EuMC '11), pp. 53-56, Manchester, UK, 2011.

[32] A. M. Nicolson and G. F. Ross, "Measurement of the intrinsic properties of materials by time-domain techniques," IEEE Transactions on Instrumentation and Measurement, vol. 19, pp. 377-382, 1970.

[33] W. B. Weir, "Automatic measurement of complex dielectric constant and permeability at microwave frequencies," Proceedings of the IEEE, vol. 62, no. 1, pp. 33-36, 1974.

[34] S.-R. Berardi, R. Yan, M. M. Kelly et al., "Broadband graphene terahertz modulators enabled by intraband transitions," Nature Communications, vol. 3, article 780, 2012.

[35] B. Sensale-Rodriguez, Y. Rusen, L. Lei, D. Jena, and H. G. Xing, "Graphene for reconfigurable terahertz optoelectronics," Proceedings of the IEEE, vol. 101, no. 7, pp. 1705-1716, 2013.

[36] J. L. Tomaino, A. D. Jameson, J. W. Kevek et al., "Terahertz imaging and spectroscopy of large-area single-layer graphene," Optics Express, vol. 19, pp. 141-146, 2011.

[37] D.-x. Zhou, E. P. J. Parrott, D. J. Paul, and J. A. Zeitler, "Determination of complex refractive index of thin metal films from terahertz time-domain spectroscopy," Journal of Applied Physics, vol. 104, Article ID 053110, 2008.

[38] I. Maeng, S. Lim, S. J. Chae, Y. H. Lee, H. Choi, and J.-H. Son, "Gate-controlled nonlinear conductivity of Dirac fermion in graphene field-effect transistors measured by terahertz timedomain spectroscopy," Nano Letters, vol. 12, no. 2, pp. 551-555, 2012. 

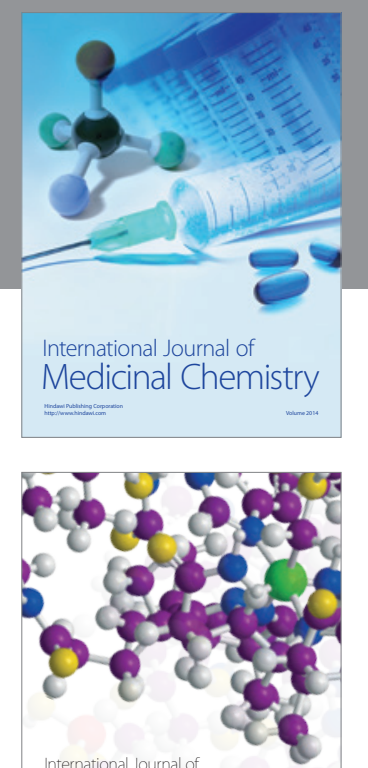

\section{Carbohydrate} Chemistry

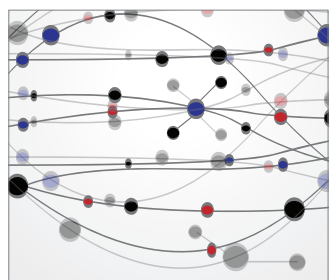

The Scientific World Journal
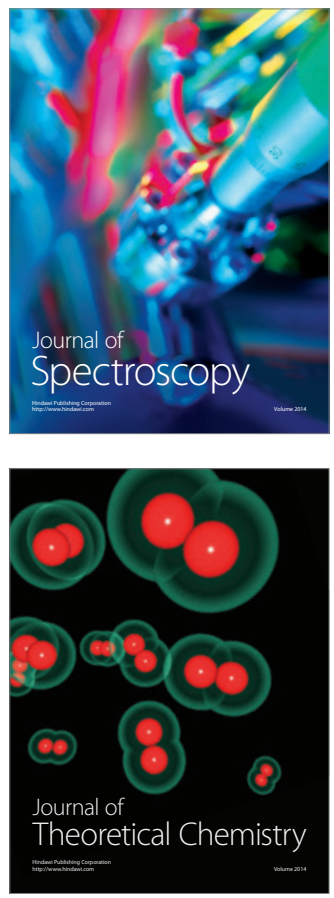
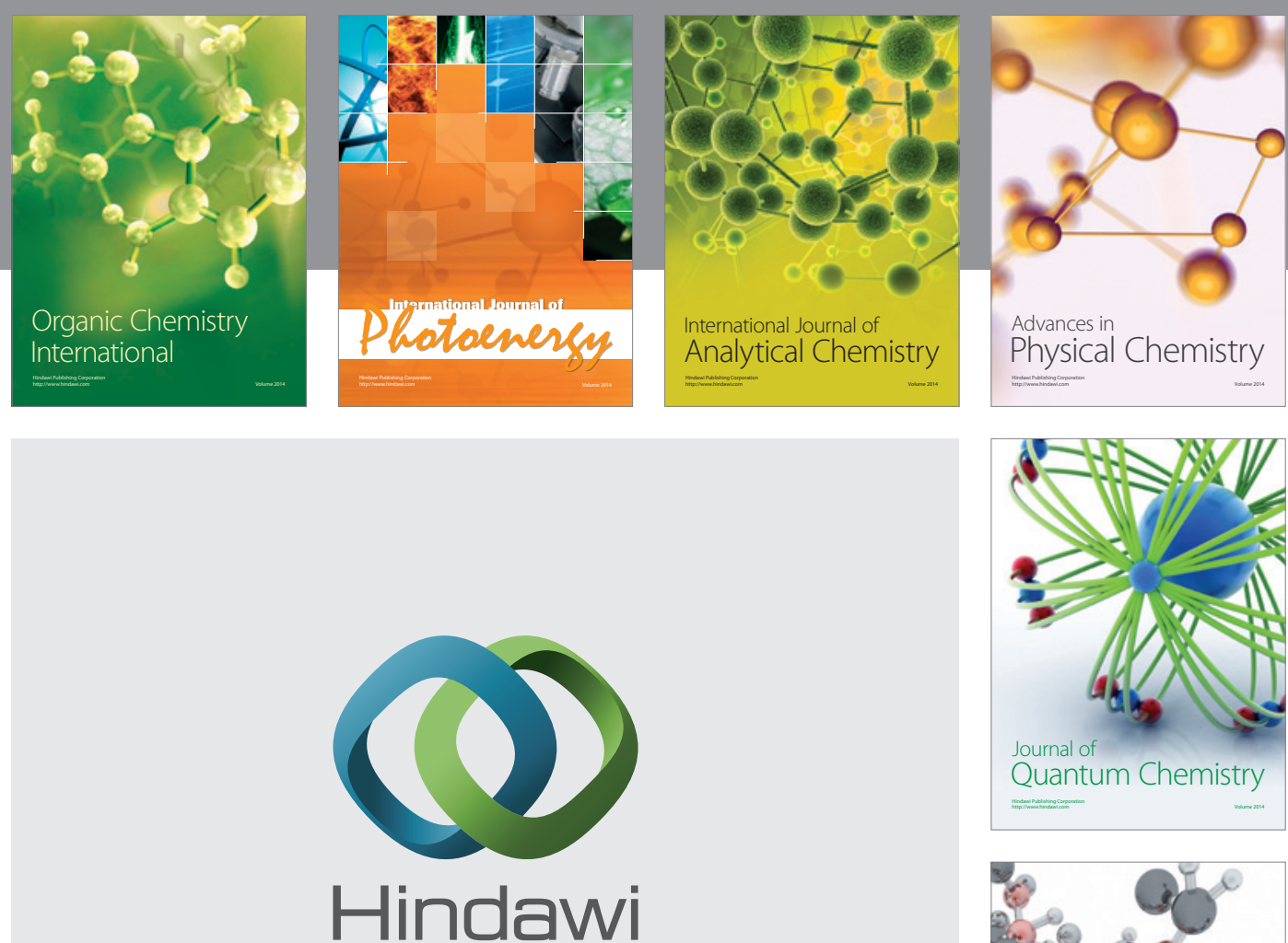

Submit your manuscripts at

http://www.hindawi.com

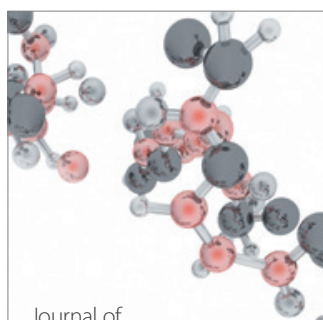

Analytical Methods

in Chemistry

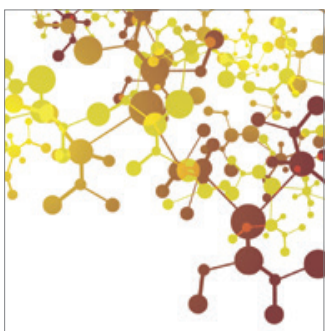

Journal of

Applied Chemistry

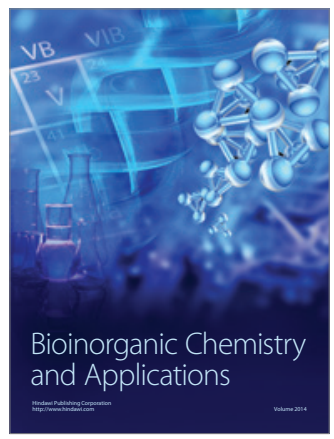

Inorganic Chemistry
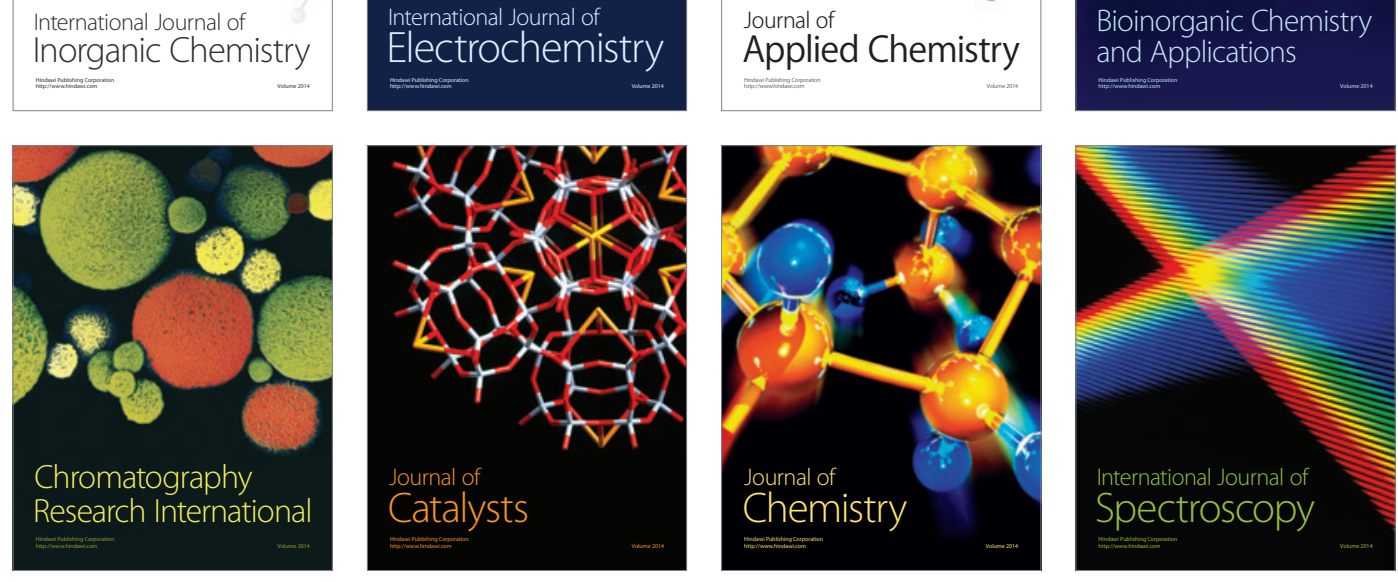